Submitted to Antrophoprical Jow Letters

\title{
Spreading of thermonuclear flames on the neutron star in SAX J1808.4-3658: an observational tool
}

\author{
Sudip Bhattacharyya ${ }^{1,2}$, and Tod E. Strohmayer ${ }^{2}$
}

\begin{abstract}
We analyse archival Rossi X-Ray Timing Explorer (RXTE) proportional counter array (PCA) data of thermonuclear X-ray bursts from the 2002 outburst of the accreting millisecond pulsar SAX J1808.4-3658. We present evidence of a complex frequency modulation of oscillations during burst rise, and correlations among the time evolution of the oscillation frequency, amplitude, and the inferred burning region area. We discuss these findings in the context of a model, based on thermonuclear flame spreading on the neutron star surface, that can qualitatively explain these features. From our model, we infer that for the 2002 Oct. 15 thermonuclear burst, the ignition likely occured in the mid-latitudes, the burning region took $\sim 0.2 \mathrm{~s}$ to nearly encircle the equatorial region of the neutron star, and after that the lower amplitude oscillation originated from the remaining asymmetry of the burning front in the same hemisphere where the burst ignited. We emphasize that studies of the evolution of burst oscillation properties during burst rise can provide a powerful tool to understand thermonuclear flame spreading on neutron star surfaces under extreme physical conditions.
\end{abstract}

Subject headings: equation of state - methods: data analysis - stars: neutron - X-rays: binaries - X-rays: bursts - X-rays: individual (SAX J1808.4-3658)

\section{Introduction}

X-ray bursts are produced by thermonuclear burning of matter accumulated on the surfaces of accreting neutron stars (Grindlay et al. 1976; Belian, Conner, \& Evans 1976; Woosley, \& Taam 1976, Joss 1977; Lamb, \& Lamb 1978). During many bursts, millisecond period brightness oscillations are generated by the combination of rapid stellar rotation

\footnotetext{
${ }^{1}$ Department of Astronomy, University of Maryland at College Park, College Park, MD 20742-2421

${ }^{2}$ X-ray Astrophysics Lab, Exploration of the Universe Division, NASA's Goddard Space Flight Center, Greenbelt, MD 20771; sudip@milkyway.gsfc.nasa.gov, stroh@clarence.gsfc.nasa.gov
} 
and an asymmetric brightness pattern on the neutron star surface (Strohmayer, \& Bildsten 2003). The period of these oscillations is very close to the stellar spin period (Chakrabarty et al. 2003; Strohmayer et al. 2003). Moreover, as this timing feature originates from the surface of the neutron star, its detailed modeling may be useful to constrain stellar structure parameters, and hence the equation of state models of the dense matter in the neutron star core (Bhattacharyya et al. 2005; Miller, \& Lamb 1998; Nath, Strohmayer, \& Swank 2002; Muno, Özel, \& Chakrabarty 2002).

Modeling of burst oscillations can also be useful to understand neutron star atmospheres, surface fluid motions, and for mapping the magnetic field structure on the stellar surface. For example, the evolution of frequency of these oscillations during the burst rise phase, may provide information on the spreading of thermonuclear flames under the extreme physical conditions that exist on neutron stars (Bhattacharyya \& Strohmayer 2005b). This is because bursts almost certainly ignite at a particular point on the stellar surface (as simultaneous ignition over the whole surface would require very fine tuning), and then spread to burn all the surface fuel (Spitkovsky, Levin, \& Ushomirsky 2002; Bhattacharyya \& Strohmayer 2005a). This slow (compared to the rotational speed) movement and spreading of the burning region may give rise to complex frequency evolution of the observed burst oscillations. This spreading would also cause the observed burst intensity to increase, and the oscillation amplitude to decrease. Moreover, the increase in emission area can be estimated by spectral analysis (Strohmayer, Zhang \& Swank 1997; Bhattacharyya \& Strohmayer 2005a; 2005c). Therefore, simultaneous modeling of the evolution of burst intensity, oscillation frequency and amplitude, and spectral properties can, in principle, be a powerful tool to understand the propagation of burning fronts on neutron star surfaces under conditions of extreme radiative pressure, magnetic field and gravity.

Frequency evolution during burst rise oscillations has so far been observed from two low mass X-ray binary (LMXB) sytems: SAX J1808.4-3658 and 4U 1636-536 (Chakrabarty et al. 2003; Bhattacharyya \& Strohmayer 2005b). The Rossi X-ray Timing Explorer (RXTE) observed the $401 \mathrm{~Hz}$ X-ray pulsar SAX J1808.4-3658 in October and November of 2002, when it was in outburst. Four type I X-ray bursts were detected during these observations (Chakrabarty et al. 2003), three of which showed strong millisecond period brightness oscillations during burst rise. A previous study found that as the burst intensity rises, the oscillation frequency also increases by $\sim 5 \mathrm{~Hz}$ and may overshoot the stellar spin frequency (Chakrabarty et al. 2003). Here, we analyse these archival data in order to model the time evolution of different burst properties and search for correlations among them.

In our study we find evidence of complex variations in the oscillation frequency during the rising phase of bursts. This is the first report of such variations from any source. The 
frequency modulation is correlated with the evolution of oscillation amplitude and burning region area. In $\S 3$, we propose a qualitative model, based on thermonuclear flame propagation, that can explain the evolution of different burst properties, as well as the correlations among them.

\section{Data Analysis and Results}

We analyse the archival RXTE proportional counter array (PCA) data of the 2002 outburst from SAX J1808.4-3658. Three thermonuclear bursts with significant millisecond oscillations during the rising phase are found in the Obslds: 70080-01-01-000 (Oct 15), 7008001-02-000 (Oct 18), and 70080-01-02-04 (Oct 19). First we explore the frequency evolution of burst rise oscillations during these bursts using three procedures. We calculate dynamic power spectra (Strohmayer \& Markwardt 1999) with time sampling short enough to resolve the burst rise interval, but large enough to accumulate sufficient signal power above the noise level. The dynamic spectra (panel $a$ of Fig. 1, and Fig. 2) provide indications of the frequency evolution behavior. To confirm the indications in the dynamic spectra, we carry out a phase timing analysis (Muno et al. 2000). We divide the burst rise time interval into several bins of a fixed chosen length, and then assuming a frequency evolution model, we calculate the average phase $\left(\psi_{k}\right)$ in each bin $(k)$. The corresponding $\chi^{2}$ is calculated using the formula $\chi^{2}=\sum_{k=1}^{M}\left(\psi_{k}-\bar{\psi}_{k}\right)^{2} / \sigma_{\psi_{k}}^{2}$ (Strohmayer \& Markwardt 2002), where $M$ is the number of bins. For this study we used extensive burst rise simulations to evaluate the uncertainty, $\sigma_{\psi_{k}}$, as a function of the $Z^{2}$ power in each time bin. We find the best fit parameter values for various frequency evolution models by minimizing $\chi^{2}$, and we calculate the uncertainty in each parameter by finding the change which produces the appropriate increase in $\chi^{2}$ (Strohmayer \& Markwardt 2002; Press et al. 1992). Finally, we calculate the total $Z^{2}$ power (Strohmayer \& Markwardt 2002) for the entire rise interval (ie. without binning) using the best fit frequency evolution model parameters, and ensure that this power is close to the maximum power obtained from any parameter values.

We first fit the oscillations during the rising phase of the Oct. 15 burst with a constant frequency model. This gives a best fit frequency of $400.91 \mathrm{~Hz}$ and $\chi^{2} /$ dof $=30.04 / 8$. We evaluate the significance of this $\chi^{2}$ value using simulations, and find a probability of 0.005 to obtain such a value by chance. Since the constant frequency model does not describe the data well, we next consider more complex models. These are; (1) linear frequency increase, (2) second order polynomial, and (3) linear increase and subsequent linear decrease models (see Table 1 for a description of the models). These models give $\chi^{2} /$ dof values of $17.73 / 7$, $17.35 / 6$, and $11.68 / 5$ respectively. Although these models give better fits, they still have 
reduced $\chi^{2}$ values $>2$, and do not describe the data very well. A slightly more complex model which fits the data well has a linear frequency increase followed by a second order polynomial, and gives a $\chi^{2} /$ dof value of 3.36/4. Of the models tested we consider this the best description of the Oct. 15 burst, and the best fit parameter values are given in Table 1. Panel $a$ of Fig. 1 shows that this model is consistent with the dynamic power contours, and it indicates a frequency increase (by a few Hertz) for the first $\sim 0.2 \mathrm{~s}$ from burst onset, then a frequency decrease (by $\sim 1 \mathrm{~Hz}$ ), and a subsequent increase. From Table 1, we note that the model parameters $\nu_{0} \& \dot{\nu}_{1}$ are practically unconstrained from the lower and upper sides respectively. This implies that the initial frequency increase can be well fit by a very steep model. However, the other sides of these parameters are reasonably well constrained, which shows that a constant frequency model is insufficient to model this portion.

Next we fit the rise oscillations from the Oct. 18 \& Oct. 19 bursts with the same frequency evolution models. The constant frequency model for the Oct. 18 burst gives a $\chi^{2} /$ dof $=154.51 / 8$, and hence can be strongly rejected. This confirms the conclusions of Chakrabarty et al. (2003) who first noted the large frequency increase present during this burst. A linear frequency increase model gives a $\chi^{2} /$ dof $=19.35 / 7$ that, though better, is still uncomfortably large to be acceptable. The next more complex model (a constant frequency, $\&$ subsequent linear increase) gives a $\chi^{2} /$ dof $=8.44 / 6$ (see Table 1 , and the upper panel of Fig. 2), and is statistically acceptable. However, we note that the dynamic power contours (upper panel, Fig. 2) are suggestive of an initial frequency increase, decrease, and increase behavior (shown by the dotted curve) qualitatively similar to that for the Oct. 15 burst. Although higher signal to noise ratios per time bin would likely be required to confirm this behavior, this (dotted curve) model does give a higher total $Z^{2}$ power than that given by the best fit $\chi^{2}$ model (solid curve) in Table 1. Finally, the constant frequency model for the Oct. 19 burst gives a $\chi^{2} /$ dof $=22.03 / 8$, which is also unacceptably high. A linear frequency increase model (see Table 1 , and the lower panel of Fig. 2), gives a $\chi^{2} /$ dof $=6.46 / 7$, and is acceptable for this burst.

Panel $b$ of Fig. 1 shows the rms amplitude variation with time during the rise of the Oct. 15 burst. From the beginning of the burst, the amplitude decreases for $\sim 0.2 \mathrm{~s}$, and then assumes a near constant value, with some fluctuations. This behavior is qualitatively similar to that seen in bursts from the LMXB systems 4U 1728-34 and 4U 1636-536 (Strohmayer, Zhang \& Swank 1997; Bhattacharyya \& Strohmayer 2005b). We also perform time resolved spectral fitting (using a blackbody model) during the rise of the Oct. 15 burst. The inferred source radius (which provides some relative indication of the size of the burning region on the stellar surface) shows evidence for a modest increase for the first $\sim 0.2 \mathrm{~s}$, and then remains almost constant (panel c, Fig. 1). Panel $d$ gives the corresponding source temperature variation. 


\section{Discussion}

Taken together our results for the three bursts from SAX J1808.4-3658 suggest that the oscillation frequency can evolve in a complex manner during burst rise. In all cases a constant frequency model is a poor description of the oscillations. In two bursts (Oct. 15 and 18) the data can best be described by a complex modulation in frequency whereby the frequency initially increases, then decreases before increasing again. We now discuss from a theoretical perspective how spreading of thermonuclear flames can plausibly account for such complex modulations in the oscillation frequency and amplitude. In our discussions we use the observational results mostly from the Oct. 15 burst as characteristic. The salient features of this burst are as follows: (1) from the start of the burst, the oscillation frequency and burning region area increase and the oscillation amplitude decreases for $\sim 0.2 \mathrm{~s} ;(2)$ after $\sim 0.2 \mathrm{~s}$, the frequency first decreases and then increases, and both amplitude and burning region area reach a nearly constant value (with some fluctuations).

The burst begins when the fuel (i.e., accumulated matter) ignites at a particular point, and then the flame propagates over the surface (Spitkovsky et al. 2002; Bhattacharyya \& Strohmayer 2005a; 2005c). Before spreading has engulfed the entire star, temperature variations due to surface waves may not be able to explain the brightness oscillation or its frequency evolution (as has been proposed for the burst tail oscillations; Heyl 2005; Lee \& Strohmayer 2005), as the rapid spreading and temperature increase may wash out this effect. This explanation of frequency evolution during burst rise was also shown to be unfavored by Bhattacharyya \& Strohmayer (2005b). However, thermonuclear flame propagation can give rise to the observed frequency evolution, as different accelerations of the eastbound and the westbound burning fronts can cause acceleration (either eastwards or westwards) of the center of the burning region relative to the star. This is our primary explanation for the observed frequency modulation. Here, we do not consider oscillations which could be caused by local vortices or magnetic fields, as even if they form, they may not necessarily generate a hot spot.

We first review some relevant results from previous work (Spitkovsky et al. 2002): (1) the greater scale height of the burning region (than the cold fuel) gives rise to a shearing speed (as the horizontal pressure gradient in the burning front increases with height). As a result, the cold fuel is drawn into the burning front and ignited. This enables the burning front to propagate. (2) The shearing speed is greater nearer the equator than the pole (due to the latitude dependence of the Coriolis parameter; Spitkovsky et al. 2002). Thus, the burning front propagates with the shearing speed $(\vartheta)$ (assuming the mixing time scale is very small; Spitkovsky et al. 2002; Fujimoto 1988; Cumming \& Bildsten 2000). Now, if the fuel ignites at a mid-latitude (say, in the northern hemisphere), it will propagate faster 
towards the equator (than the pole), and the east-west width of the burning region will increase much faster near the equator. Therefore, after a certain time, the burning region will encircle the equator and propagate more or less symmetrically towards the south pole. The northern burning front propagates towards the north pole, keeping the asymmetry (due to the variation of east-west width with latitude), which vanishes near the pole. At the beginning, the burning region is relatively small, and hence the oscillation amplitude can be large. As the burning region grows, the oscillation amplitude naturally diminishes (panel $b$ of Fig. 1). This effect was also reported for bursts from other sources (Strohmayer, Zhang, \& Swank 1997). After the burning region encircles the equator with a considerable northsouth width, the observed burning area does not increase much (hence the near constant radius after the initial increase; panel $c$ of Fig. 1). From this time, the oscillation is due to the asymmetry of the northern burning front (with the persistent background due to the azimuthally symmetric portion of the burning region), and hence the amplitude attains a near constant value till the asymmetry vanishes. Therefore, according to our explanation, for the Oct. 15 burst, the burning region takes $\sim 0.2 \mathrm{~s}$ to nearly encircle the stellar equator.

We consider two additional effects to explain the complex frequency evolution: (3) due to the increased scale height, the top portion of the burning region slips westwards (if the star rotates eastwards) to conserve angular momentum. If the shearing speed due to this is $v$, the burning front propagates westwards with a speed $v+\vartheta$ and eastwards with a speed $\vartheta$. (4) The angular momentum of a stellar surface element increases from pole to equator. Therefore, to conserve angular momentum, if the burning front propagates towards the equator, it drifts westward, and if it propagates towards the pole, it drifts eastward. The corresponding speed $(V)$ depends on latitude. For a given value of $\vartheta, V$ is larger near the pole, as the fractional change of angular momentum is larger there for a given amount of latitude change. However, $V$ is a monotonically increasing function of $\vartheta$, and $\vartheta$ increases from pole to equator. So, two competing effects determine the latitude and direction dependence (northbound and southbound) of $V$. Another effect may also be relevant for the direction dependence of $V$. At a given latitude in the northern hemisphere, if the front moves southwards, it is pulled downwards by the increase in the effective gravity (as it conserves its angular momentum), perhaps making the friction and the mixing within the front more efficient. Hence the front speed both southwards and westwards may increase, and the value of $V$ is greater. For the opposite reason, at the same latitude, a northbound front has a smaller $V$. Considering these three effects on $V$, it seems likely that in the northern hemisphere, $V$ is larger for a southbound front at low-latitudes than for a northbound front at mid-latitudes.

Our explanation for the observed frequency modulation uses all four effects discussed above. If ignition starts at a mid-latitude in the northern hemisphere and initially propagates mostly southwards, its eastbound front has a speed $\vartheta$, while the westbound front has speed 
$V+v+\vartheta$ (the star rotates eastwards). Therefore, as $\vartheta$ is likely to be smaller than $V+v$ in the mid-latitudes, the observed frequency starts from a value $\sim \mathrm{a}$ few $\mathrm{Hz}$ lower than the stellar spin frequency. As the front approaches the equator, $\vartheta$ increases quickly, and as it crosses the equator, the term $V$ switches from westbound to eastbound. These two effects quickly bring the observed frequency back towards the stellar spin frequency. Indeed, if $V$ is large enough then it may cause the observed frequency to overshoot the spin frequency. This goes on till the burning region more or less encircles the star around the equator. The fact that the peak frequency occurs $\sim 0.2 \mathrm{~s}$ (panel $a$ of Fig. 1) after the beginning of the burst supports this qualitative scenario. After the equatorial belt has ignited, the oscillation results from the remaining asymmetry of the northern front. The westward speed of the west front of this asymmetry is $v+\vartheta$, while the eastward speed of its east front is $V+\vartheta$. The competition between these two speeds determines the frequency evolution. If initially, during the time interval when this asymmetry takes over, $v>V$ (in the mid-latitudes), then the observed frequency decreases, and then, as $V$ eventually dominates (as the front proceeds northwards), the observed frequency starts to increase again, and may, in fact, overshoot the stellar spin frequency. This can explain the frequency evolution of the Oct. 15 burst (panel $a$, Fig. 1). However, if $V>v$ immediately after the equatorial belt is ignited, the oscillation frequency increases monotonically (lower panel of Fig. 2; also Fig. 1 \& Fig. 2 of Bhattacharyya \& Strohmayer 2005b). We argue that the Oct. 15 burst was ignited at a comparatively lower latitude, and hence $v$ was greater than $V$ for some time after equatorial belt ignition, while the Oct 19 burst and the bursts from 4U 1636-536 (that showed burst rise frequency evolution; Bhattacharyya \& Strohmayer 2005b) were ignited at slightly higher latitudes.

In their study of thermonuclear burning on rotating neutron stars Spitkovsky et al. (2002) derived the flame speed formula, $\nu_{\text {flame }}=(g h)^{1 / 2} / f t_{n}$, where $g, h, f$ and $t_{n}$ are the surface gravity, burning layer scale height, Coriolis parameter $\left(f=2 \Omega_{\mathrm{spin}} \cos \theta\right)$, and nuclear burning timescale, respectively. An interesting question is whether the spin frequency dependence can be tested by comparing oscillation properties in bursts from different sources. Although one might expect such properties as burst rise times to reflect the flame speed, other time scales, such as the radiative diffusion time scale, are also relevant for rise times, and could mask the front speed effects. A perhaps more robust indication of the flame speed could be the time scale over which the oscillation amplitude drops during the early rising phase. Initial indications are that the amplitude drops more quickly in the Oct. 15 burst from SAX J1808.4-3658 than a burst from $4 \mathrm{U}$ 1636-536 that also shows frequency evolution during the rising phase (see Bhattacharyya \& Strohmayer 2005b). At face value this is consistent with the spin frequency dependence from Spitkovsky et al. (2002), that is, other things being equal, flames propagate more slowly on more rapidly spinning stars. 
However, this is still just suggestive, as it is difficult to be precise based on only several bursts. Nevertheless, it seems clear that further studies of burst rise frequency evolution from a larger sample of bursts may provide important statistical information about how the flame speed and other flame spreading properties can be influenced by stellar spin, compactness, and burst strength. Therefore, we emphasize that such studies provide a potentially powerful tool to understand thermonuclear flame spreading on neutron star surfaces under extreme physical conditions.

This work was supported in part by NASA Guest Investigator grants. 


\section{REFERENCES}

Belian, R. D., Conner, J. P., \& Evans, W. D. 1976, ApJ, 206, L135.

Bhattacharyya, S., \& Strohmayer, T. E. 2005a, ApJ, in press (astro-ph/0509369).

Bhattacharyya, S., \& Strohmayer, T. E. 2005b, ApJ, 634, L157 (astro-ph/0509370).

Bhattacharyya, S., \& Strohmayer, T. E. 2005c, in preparation.

Bhattacharyya, S., Strohmayer, T. E., Miller, M. C. \& Markwardt, C. B. 2005, ApJ, 619, 483.

Chakrabarty, D. et al. 2003, Nature, 368, 42.

Cumming, A. \& Bildsten, L. 2000, ApJ, 544, 453.

Fujimoto, M. Y. 1988, A\&A, 198, 163.

Grindlay, J. E. et al. 1976, ApJ, 205, L127.

Heyl, J. S. 2005, MNRAS, 361, 504.

Joss, P. C. 1977, Nature, 270, 310.

Lamb, D. Q., \& Lamb, F. K. 1978, ApJ, 220, 291.

Lee, U., \& Strohmayer, T. E. 2005, MNRAS, 361, 659.

Miller, M. C. \& Lamb, F. K. 1998, ApJ, 499, L37.

Muno, M. P., Fox, D. W., Morgan, E. H. \& Bildsten, L. 2000, ApJ, 542, 1016.

Muno, M. P., Özel, F., \& Chakrabarty, D. 2003, ApJ, 595, 1066.

Nath, N. R., Strohmayer, T. E. \& Swank, J. H. 2002, ApJ, 564, 353.

Press, W. H., Teukolsky, S. A., Vetterling, W. T., \& Flannery, B. P. 1992, Numerical Recepies in FORTRAN (New York: Cambridge University Press), 687-693.

Spitkovsky, A., Levin, Y. \& Ushomirsky, G. 2002, ApJ, 566, 1018.

Strohmayer, T. E., \& Bildsten, L. 2003, in Compact Stellar X-ray Sources, Eds. W.H.G. Lewin and M. van der Klis, (Cambridge University Press: Cambridge), (astroph/0301544). 
Strohmayer, T. E. \& Markwardt, C. B. 1999, ApJ, 516, L81.

Strohmayer, T. E. \& Markwardt, C. B. 2002, ApJ, 577, 337.

Strohmayer, T. E., Markwardt, C.B., Swank, J. H., \& in 't Zand, J. 2003, ApJ, 596, L67.

Strohmayer, T. E., Zhang, W. \& Swank, J. H. 1997, ApJ, 487, L77.

Woosley, S. E., \& Taam, R. E. 1976, Nature, 263, 101. 
Table 1. Frequency evolution model parameters ${ }^{2}$ (with $1 \sigma$ error) for burst rise oscillations of three bursts from SAX J1808.4-3658.

\begin{tabular}{cccccccc}
\hline \hline Burst date & $\nu_{0}$ & $\dot{\nu}_{1}$ & $t_{\mathrm{b}_{1}}$ & $\dot{\nu}_{2}$ & $\ddot{\nu}_{2}$ & $\chi^{2} /$ dof & $Z_{1}^{\text {2b }}$ \\
\hline 2002 Oct 15 & $396.72_{-21.73}^{+2.00}$ & $29.64_{-13.95}^{+236.21}$ & $0.20_{-0.10}^{+0.05}$ & $-15.54_{-4.94}^{+4.73}$ & $23.67_{-9.81}^{+11.52}$ & $3.36 / 4$ & 152.16 \\
2002 Oct 18 & $398.22_{-0.21}^{+0.20}$ & - & $0.31_{-0.04}^{+0.04}$ & $19.21_{-3.39}^{+5.19}$ & - & $8.44 / 6$ & 62.83 \\
2002 Oct 19 & $399.64_{-0.14}^{+0.23}$ & $1.69_{-0.42}^{+0.07}$ & - & - & - & $6.46 / 7$ & 86.64 \\
\hline
\end{tabular}

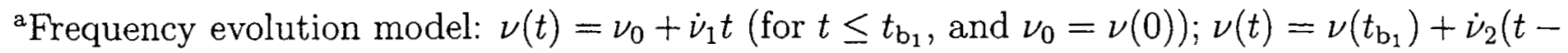
$\left.t_{\mathrm{b}_{1}}\right)+\ddot{\nu}_{2}\left(t-t_{\mathrm{b}_{1}}\right)^{2}$ (for $t \geq t_{\mathrm{b}_{1}}$ ).

bFundamental power during burst rise. 


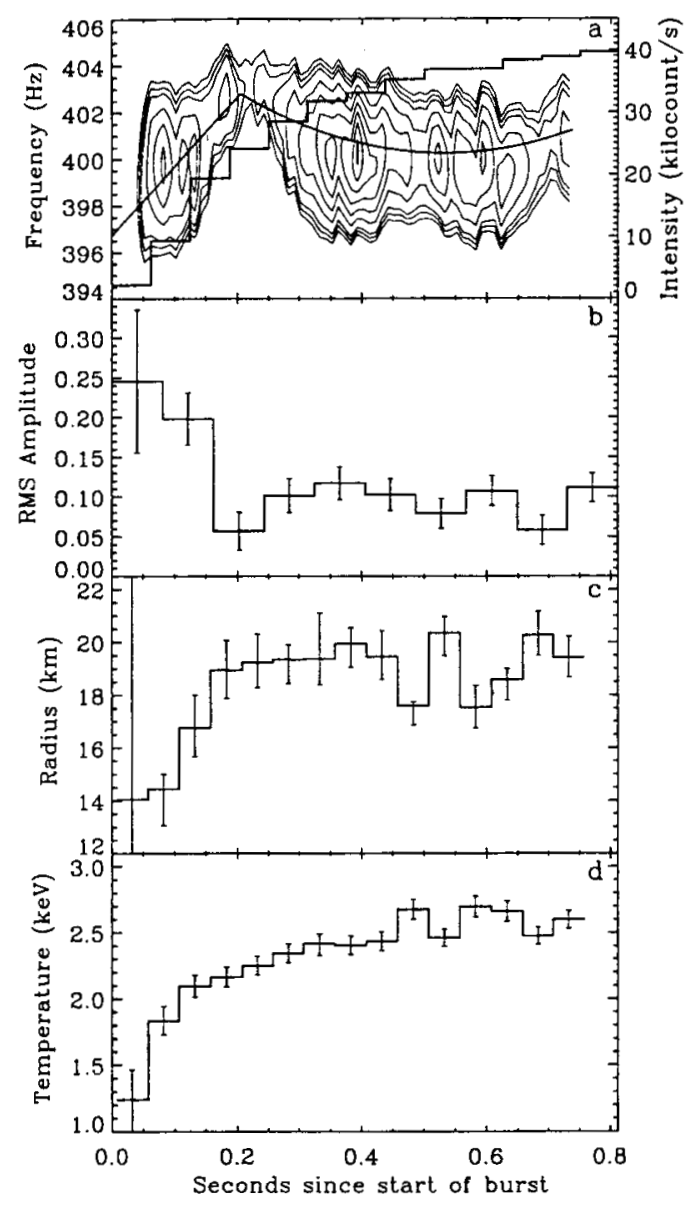

Fig. 1.- Time evolution of different observed burst properties during the rise of the Oct 15 burst from SAX J1808.4-3658. Panel $a$ gives the detected intensity (histogram), power contours (minimum and maximum power values are 16 and 51) from the dynamic power spectra (for $0.15 \mathrm{~s}$ duration at $0.01 \mathrm{~s}$ intervals), and the best fit model from Table 1 . Panel $b$ shows the rms amplitude of the oscillations. Here the horizontal lines give the binsize. Panel $c$ gives the inferred radius (assuming $10 \mathrm{kpc}$ source distance) of the source, while panel $d$ gives the corresponding temperature. For panels $b, c$, and $d$, persistent emission is subtracted and a deadtime correction is applied, and the error bars are $1 \sigma$ values. 

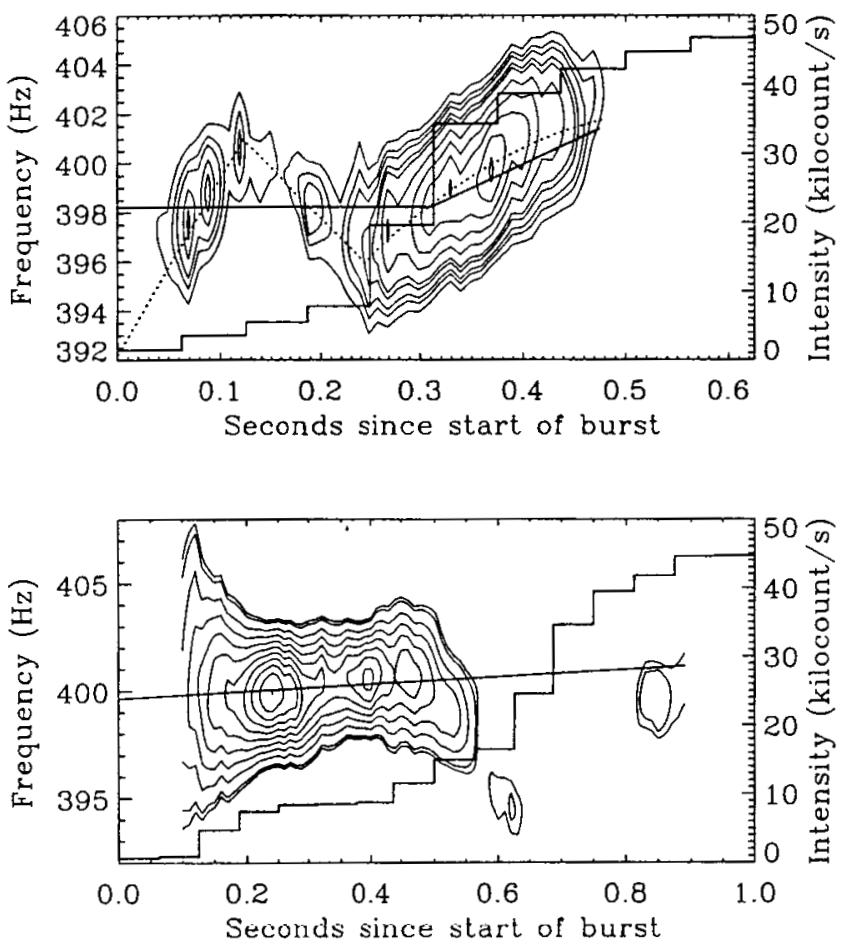

Fig. 2.- Similar to panel $a$ of Fig. 1 but for the Oct 18 (upper) and Oct 19 (lower) bursts. For the upper panel dynamic power spectra are calculated for $0.15 \mathrm{~s}$ duration at $0.01 \mathrm{~s}$ intervals, while for the lower panel these numbers are $0.2 \mathrm{~s}$ and $0.01 \mathrm{~s}$. For the calculation of power contours, minimum and maximum values of power are 15 and 50 for the upper panel, and 17 and 111 for the lower panel. The dotted line in the upper panel gives the frequency evolution for which the total power (74) is higher than that (63) corresponding to the best fit model (solid line) from Table 1. 